\section{Predisposed genomic instability in pre-treatment bone marrow evolves to therapy-related myeloid neoplasms in malignant lymphoma}

Therapy-related myeloid neoplasm (tMN) occurs as a late complication of cytotoxic chemotherapy and/or radiation therapy. Approximately $30 \%$ of all tMN occur in patients with a previous hematologic malignancy, mostly malignant lymphomas (ML). ${ }^{1} \mathrm{ML}$ patients are likely to receive multiple types of chemotherapy and hence, the underlying developmental mechanism of $\mathrm{tMN}$ remains elusive. ${ }^{1}$

Clonal somatic mutations, detectable in myelodysplastic syndrome (MDS) or acute myeloid leukemia (AML), are found in a subset of aged healthy individuals and are collectively termed as clonal hematopoiesis of indeterminate potential (CHIP)., ${ }^{2,3}$ Among such individuals with CHIP in their peripheral blood (PB), some develop hematologic neoplasia later. ${ }^{2}$ Certain mutations in CHIP, as in $D N M T 3 A$ and TET2, overlap with the somatic mutations found in MDS patients, therefore, CHIP is considered a step towards MDS. ${ }^{3}$ In previous reports, clonal hematopoiesis was found in $\mathrm{PB}$ samples in $\mathrm{tMN}$ before chemotherapy. ${ }^{4-7}$ Moreover, stored hematopoietic stem cell samples from tMN patients prior to autologous hematopoietic stem cell transplantation had clonal hematopoiesis with detectable preleukemic mutations, ${ }^{8,9}$ while most tMN patients received chemotherapy before the collection of autologous stem cells. Therefore, it is still uncertain if: (1) clonal hematopoiesis present in the bone marrow (BM) before any therapy could be responsible for $\mathrm{tMN}$; and (2) clonal hematopoiesis, if detectable prior to any therapy, could emerge and evolve into a tMN clone. We conducted a molecular analysis using myeloid targeted deep sequencing in pre-treatment and tMN BM samples.

Among $494 \mathrm{ML}$ patients (median age, 65 years) enrolled at Tokyo Medical University between 2008 and 2018, six had $\mathrm{tMN}$ onset during the post-treatment follow-up. BM samples from five patients (unique patient number: UPN 40, 47-50) were obtained at the time of $\mathrm{ML}$ diagnosis and $\mathrm{tMN}$ onset. $\mathrm{BM}$ involvement of $\mathrm{ML}$ cells was not found by pathological analysis or flow cytometry in any of the patients. The median duration from ML diagnosis to $\mathrm{tMN}$ diagnosis was 48 months (range: 24-96 months). We analyzed BM mononuclear cells (BMMC) collected at both $\mathrm{ML}$ and $\mathrm{tMN}$ diagnoses by myeloid targeted deep sequencing. BMMC collected at the time of diagnosis of idiopathic thrombocytopenic purpura (ITP), 6 years before the ML diagnosis, were available for UPN 49 and were used in the analysis of $\mathrm{tMN}$ development in this patient. To determine whether $\mathrm{ML}$ and tMN cells had consistent mutations, we also used ML tissues from these patients.

BMMC from four patients treated for ML who did not develop tMN were used as controls. These control patients had received standard or intensive chemotherapy and had been followed for longer than the median follow-up duration of $\mathrm{tMN}$ group (median observation period after ML diagnosis was 60 months; range: 48-84 months). Written informed consent was obtained from each patient. The study was approved by the institutional review board of Tokyo Medical University (approval no. 2,151$)$.

The median sequence depth was 1,790 coverage in this study (Online Supplementary Figure S1). The analysis enabled us to detect cytogenetic abnormalities or preleukemic mutations related to myeloid malignancy in
4 (UPN 47-50) of 5 tMN cases. Conversely, all four ML control subjects had normal karyotypes; none of them had any significant preleukemic mutations (Online Supplementary Table S1).

In the four tMN cases with significant preleukemic mutations, we observed no difference in the number of non-synonymous mutations detected in the BM samples at the time of $\mathrm{ML}$ and $\mathrm{tMN}$ diagnoses (medians, 15 and 14, respectively). Except for UPN49, in which was revealed that the main clone of clonal hematopoiesis in pre-treatment $\mathrm{BM}$ at the time of $\mathrm{ML}$ diagnosis was undetectable in $\mathrm{BM}$ at $\mathrm{tMN}$ diagnosis.

In UPN 47, we observed cytogenetic abnormalities in chromosomes 17 and 18, which were considered as clonal mosaicism ${ }^{10,11}$ in the BM at the time of diagnosis of diffuse large B-cell lymphoma (DLBCL). However, at the time of $\mathrm{tMN}$ diagnosis, clones harboring a complex karyotype and ZRSR2 R290* mutation had expanded into a dominant clone (Figure 1A).

In UPN 48, a small clone with NRAS Q61L, DNMT3A W753*, and TP53 T125M mutations was detected in the $\mathrm{BM}$ at the time of diagnosis of DLBCL. However, undetected clones with the TP53 R280S mutation had newly emerged and expanded with an additional TET2 R83* mutation at the time of tMN diagnosis (Figure 1B).

In UPN 49, a clone with TET2 R1359S mutation and a clone with TET2 D545Tfs*10 mutation in addition to RUNX1 R201* mutation were detected in the BM at the time of ITP diagnosis, which was 6 years prior to the diagnosis of nodular sclerosis classical Hodgkin lymphoma (NSCHL). At the time of NSCHL diagnosis, the TET2 R1359S clone had acquired an additional NRAS Y64D mutation and had expanded as a dominant clone of tMN with an additional NF1 L1015Q mutation (Figure 1C). Morphological and cytogenetic abnormalities in BM were not detected in samples collected either 6 years prior to or at the time of NSCHL diagnosis.

In UPN 50, a clone with the DNMT3A V567del mutation was detected as the main clone in the BM at the time of DLBCL diagnosis. However, a clone with the SF3B1 K666N mutation, which was also detected as a minor clone at the time of DLBCL diagnosis, acquired TP53 R156_A159dup and RUNX1 S141* mutations and evolved as a dominant clone in BM at the time of tMN diagnosis (Figure 1D).

Sequential clonal evolution was not confirmed in one tMN patient (UPN 40). We speculated that $\operatorname{inv}(16)(\mathrm{p} 13 ; \mathrm{q} 22)$ was responsible for the onset of $\mathrm{tMN}$ in UPN 40. Also, no common mutations with tMN were detected in ML tissue.

Two large studies reported by Gills et al. and Takahashi et al. showed that patients with clonal hematopoiesis in pre-treatment $\mathrm{PB}$ samples were significantly likely to develop tMN after treatment. ${ }^{5,6}$ Furthermore, Takahashi et al. reported that chromosome arm-level copy number alterations detected as clonal hematopoiesis in PB samples were at risk of developing $\mathrm{tMN}^{7}{ }^{7}$ In the current study, $80 \%$ of tMN patients showed cytogenetic abnormalities or preleukemic mutations related to myeloid malignancy in the BM before chemotherapy for ML, while none of those without clonal genomic changes at the time of ML diagnosis developed tMN. In UPN 47 and UPN 48, preleukemic mutations were detected at the time of ML diagnosis, however these mutations were replaced by undetected clones at the time of ML diagnosis and developed to tMN. Of note, in UPN 50 (Figure 1 D), a minor clone carrying $S F 3 B 1$ mutation became a major tMN clone, instead of a DNMT3A-bearing clone at $\mathrm{ML}$ diagnosis. Therefore, these $\mathrm{tMN}$ patients harbored 
clonal hematopoiesis before ML therapy, but exhibited clonal changes during ML chemotherapy. In UPN 49 (Figure 1C), clones with RUNX1 R201* or TET2 R1359S had already existed at 6 years before the ML diagnosis. Although we could not obtain a germline control in this case, RUNX1 R201* has been reported as a germline mutation in familial platelet disorder with associated myeloid malignancy (FPDMM). ${ }^{12}$ We speculated that UPN 49 was likely FPDMM and had a germline predisposition to develop tMN. The current study demonstrated that: (1) tMN patients before ML chemotherapy frequently exhibit genomic changes, such as clonal hematopoiesis or germline predisposition in the BM; and (2) mutations that evolve into tMN are often undetectable at the time of ML diagnosis. Thus, we speculate that patients who develop tMN may carry many detectable or undetectable genetic changes at the time of ML diagnosis, irrespective of the clonal size. The presence of clonal hematopoiesis before ML chemotherapy might represent a predisposition to generate multiple

A

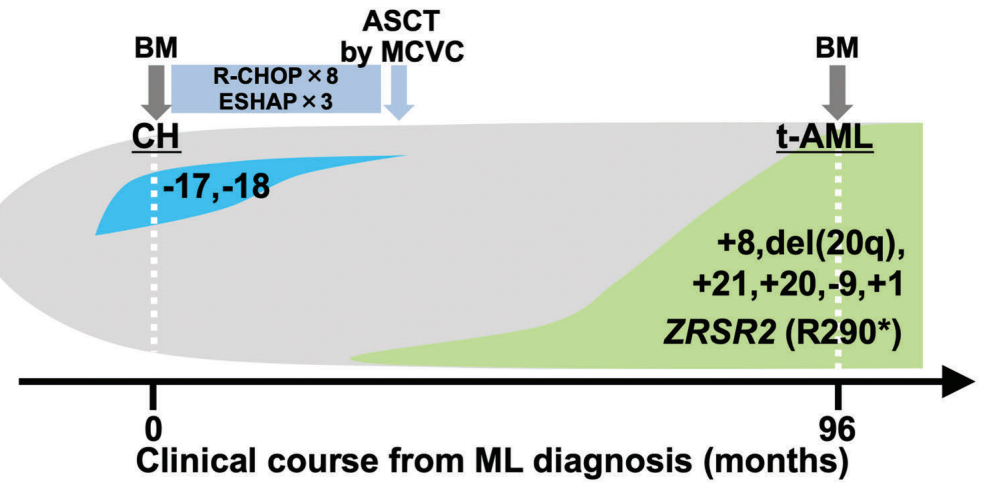

B

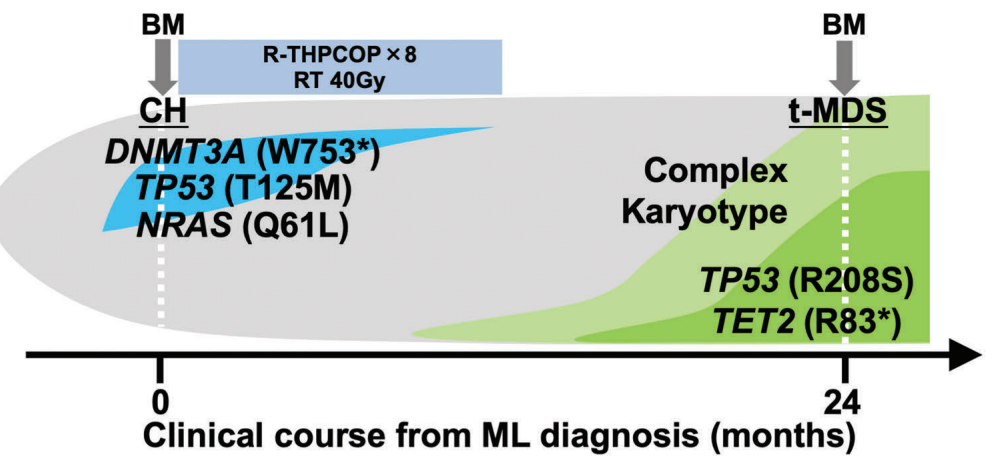

C

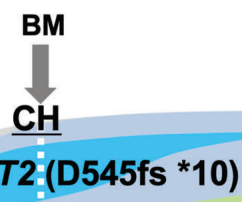

RUNX1

(R201*)

TET2

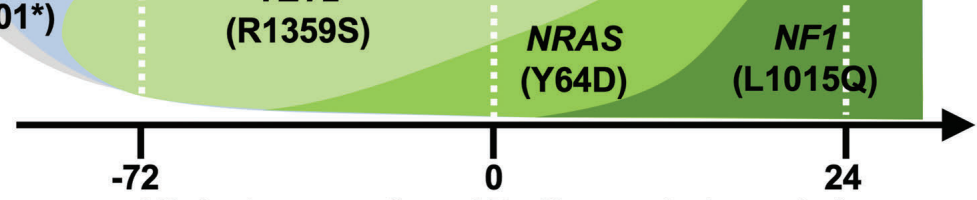

Clinical course from ML diagnosis (months)

D

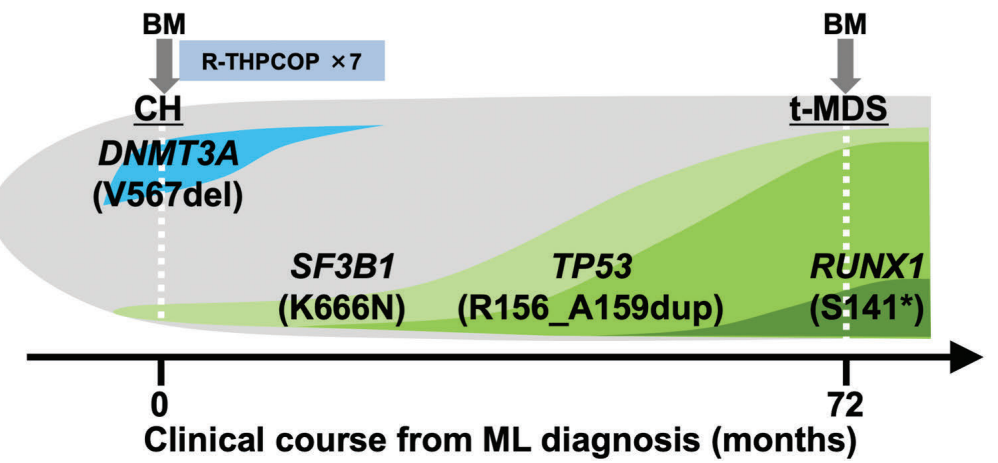

Figure 1. Graphical representation of clonal evolution of therapy-related myeloid neoplasm patients. Genomic DNA extraction from BMMC and Gene Read DNAseq library preparation was performed as reported previously. ${ }^{13}$ Genomic DNA was extracted from three formalin-fixed paraffin-embedded samples of ML tissue from each patient using a GeneRead DNA FFPE Kit (Qiagen, Hilden, Germany). Targeted DNA sequencing with a Myeloid Neoplasms Panel (Qiagen) including 50 known or putative hematopoietic mutation gene targets was carried out using a MiSeq sequencer (Illumina, San Diego, CA, USA). Sequencing reads were mapped and annotated using the Qiagen cloud-based mapping and annotating tool, GeneRead Panel Variant Calling. High probability oncogenic genetic variants were extracted from annotated data based on ClinVar (https://www.ncbi.n/m.nih.gov/ clinvar/) and COSMIC (http://cancer.sanger.ac.uk/cosmic) databases. Adopted variants were extracted with a coverage of $\geq 200$ and a variant allele frequency of $\geq 0.02$. Clonal preleukemic mutations are represented in italic font. In six of these mutations, the validation sequencing was performed by targeted-capture sequencing as reported previously ${ }^{14,15}$ and showed a positive predictive value of $100 \%$. In Figure $1 \mathrm{~B}$, the clonal composition of three mutations detected at the time of $\mathrm{ML}$ diagnosis was not necessarily clear. BM: bone marrow; BMMC: BM mononuclear cells; ML: malignant lymphoma; $\mathrm{CH}$ : clonal hematopoiesis; t-AML: therapy-related acute myeloid leukemia; tMDS: therapy-related myelodysplastic syndromes; R-CHOP: rituximab, cyclophosphamide, doxorubicin, vincristine, prednisolone; R-THPCOP: rituximab, cyclophosphamide, pirarubicin, vincristine, prednisolone; ABVD: doxorubicin, bleomycin, vinblastine, dacarbazine; ESHAP: etoposide, methylprednisolone, cytarabine, cisplatin; MCVC: ranimustine, carboplatin, etoposide, cyclophosphamide; ASCT: autologous hematopoietic stem cell transplantation; $\mathrm{RT}$ : radiation therapy. 
clones. Therefore, ML chemotherapy facilitates clonal selection and expansion with certain mutations. None of the control ML patients without $\mathrm{tMN}$ development showed mutations such as clonal hematopoiesis prior to chemotherapy. Genomic changes prior to chemotherapy for malignancy do not directly predict the clones that expand and link to tMN. Nevertheless, myeloid panel deep sequencing prior to chemotherapy could inform us of unstable genomic conditions and $\mathrm{tMN}$ development.

Seiichiro Katagiri, ${ }_{1}$ Hideki Makishima, ${ }^{2}$ Kenko Azuma, ${ }^{3,4}$ Yasuhito Nannya, ${ }^{2}$ Yuu Saitoh, ${ }^{1}$ Seiichiro Yoshizawa, ${ }^{1}$ Daigo Akahane, ${ }^{1}$ Hiroaki Fujimoto, ${ }^{1}$ Yoshikazu Ito, Ravi Velaga, Tomohiro Umezu, 1,5 Junko H. Ohyashiki, Seishi Ogawa ${ }^{2,6,7}$ and Kazuma Ohyashiki,

${ }^{1}$ Department of Hematology, Tokyo Medical University, Tokyo, Japan; ${ }^{2}$ Department of Pathology and Tumor Biology, Kyoto University, Kyoto, Japan; ${ }^{3}$ Department of Molecular Oncology, Institute of Medical Science, Tokyo Medical University, Tokyo, Japan; ${ }^{4}$ Current address: Tokyo Women's Medical University Institute for Integrated Medical Sciences (TIIMS), Tokyo, Japan; 'Department of Advanced Cellular Therapy, Tokyo Medical University, Tokyo, Japan; 'Institute for the Advanced Study of Human Biology (WPI-ASHBi), Kyoto University, Kyoto, Japan and 'Department of Medicine, Centre for Hematology and Regenerative Medicine, Karolinska Institute, Stockholm, Sweden

Correspondence: SEIICHIRO KATAGIRI - patchsei@yahoo.co.jp doi:10.3324/haematol.2019.229856

Funding: the authors would like to thank the Platform Project for Practical Research for Innovative Cancer Control from the Japan Agency for Medical Research and Development (AMED) (\#15Ack0106073h0002) (KO: Tokyo, SO, HM; Kyoto, Japan), the Private University Strategic Research-Based Support Project (S1311016) from the Ministry of Education, Culture, Sports, Science, and Technology (Tokyo, Japan), and JSPS KAKENHI Grant Number $19 K 16811$ for financial support.

Information on authorship, contributions, and financial \& other disclosures was provided by the authors and is available with the online version of this article at WWW. haematologica.org.

\section{References}

1. Kayser S, Döhner K, Krauter J, et al. The impact of therapy-related acute myeloid leukemia (AML) on outcome in 2853 adult patients with newly diagnosed AML. Blood. 2011;117(7):2137-2145.

2. Jaiswal S, Fontanillas P, Flannick J, et al. Age-related clonal hematopoiesis associated with adverse outcomes. N Engl J Med. 2014;371(26):2488-2498.

3. Steensma DP, Bejar R, Jaiswal S, et al. Clonal hematopoiesis of indeterminate potential and its distinction from myelodysplastic syndromes. Blood. 2015;126(1):9-16.

4. Wong TN, Ramsingh G, Young AL, et al. Role of TP53 mutations in the origin and evolution of therapy-related acute myeloid leukaemia. Nature. 2015;518(7540):552-555.

5. Gillis NK, Ball M, Zhang Q, et al. Clonal haemopoiesis and therapyrelated myeloid malignancies in elderly patients: a proof-of-concept, case-control study. Lancet Oncol. 2017;18(1):112-121.

6. Takahashi K, Wang F, Kantarjian H, et al. Preleukaemic clonal haemopoiesis and risk of therapy-related myeloid neoplasms: a casecontrol study. Lancet Oncol. 2017;18(1):100-111.

7. Takahashi K, Wang F, Kantarjian H, et al. Copy number alterations detected as clonal hematopoiesis of indeterminate potential. Blood Adv. 2017;1(15):1031-1036

8. Gibson CJ, Lindsley RC, Tchekmedyian V, et al. Clonal hematopoiesis associated with adverse outcomes after autologous stem-cell transplantation for lymphoma. J Clin Oncol. 2017;35(14):1598-1605.

9. Berger G, Kroeze LI, Koorenhof-Scheele TN, et al. Early detection and evolution of pre-leukemic clones in therapy-related myeloid neoplasms following autologous SCT. Blood. 2018;131(16):18461857

10. Jacobs KB, Yeager M, Zhou W, et al. Detectable clonal mosaicism and its relationship to aging and cancer. Nat Genet. 2012;44(6):651658.

11. Laurie CC, Laurie CA, Rice K, et al. Detectable clonal mosaicism from birth to old age and its relationship to cancer. Nat Genet. 2012;44(6):642-650

12. Sood R, Kamikubo Y, Liu P. Role of RUNX1 in hematological malignancies. Blood. 2017;129(15):2070-2082.

13. Azuma K, Umezu T, Imanishi S, et al. Genetic variations of bone marrow mesenchymal stromal cells derived from acute leukemia and myelodysplastic syndrome by targeted deep sequencing. Leuk Res. 2017;62:23-28.

14. Yoshizato T, Nannya Y, Atsuta Y, et al. Genetic abnormalities in myelodysplasia and secondary acute myeloid leukemia: impact on outcome of stem cell transplantation. Blood. 2017;129(17):23472358.

15. Shiozawa Y, Malcovati L, Galli A, et al. Aberrant splicing and defective mRNA production induced by somatic spliceosome mutations in myelodysplasia. Nat Comm. 2018;9(1):3649. 